\title{
Clinical benefits for the monitoring and modulating of subconjunctival tissue following glaucoma filtration surgery
}

\author{
Dao-Yi Yu*, William Morgan, Er-Ning Su and Stephen Cringle \\ Lions Eye Institute, Centre for Ophthalmology and Visual Science, The University of Western Australia, Australia
}

\begin{abstract}
Glaucoma filtration surgery (GFS) can be defined as an attempt to lower intraocular pressure (IOP) by the surgical formation of an artificial drainage pathway from the anterior chamber to the subconjunctival space. Surgical techniques and devices to create the drainage pathway have been continuously improved since the first attempts almost 180 years ago. Filtering procedures, such as trabeculectomy or drainage implant surgery have been used extensively in the treatment of glaucoma. Glaucoma affects $>60$ million people worldwide and is the second most common cause of irreversible blindness. A minimally invasive surgical technique including a bioengineered microfistula tube, a sophisticated implanter, and an ab-interno surgical procedure was developed by our team. Such minimally invasive surgery without surgical damage to the conjunctiva will significantly reduce the inflammatory reaction and scarring process and provide more optimal surgical outcomes. It also creates an opportunity to clinically investigate the question of the consequences of the presence of aqueous humor in the subconjunctival tissue. Our patents were licensed to a startup company (AqueSys), to commercially develop the technology and AqueSys has recently been acquired by Allergan. Commercialization also creates new opportunities for scientific research to further improve surgical outcomes. An important topic has arisen related to the monitoring and modulation of the subconjunctival tissue after GFS. We would also like to examine the conjunctiva to select the most suitable surgical site before implantation. We believe that the answer to such questions could be critical for improving the outcomes of our procedure, and indeed all other GFS procedures. We describe three avenues of research that need to be addressed whilst the clinical use of this technique is expanding. The purpose of such research is to gain the best outcomes for each patient treated with our newly developed techniques.
\end{abstract}

\section{Introduction}

Glaucoma affects $>60$ million people worldwide and is the second most common cause of irreversible blindness [1]. The main modifiable risk factor for optic nerve damage in primary open-angle glaucoma is increased intraocular pressure (IOP), thought to be primarily due to decreased outflow of aqueous humour through the trabecular meshwork and Schlemm's canal [2-5]. Surgical approaches to glaucoma treatment hope to achieve a permanent reduction in the outflow resistance and restore normal IOP with minimal complications. Many types of glaucoma surgery have been developed since the first attempts almost 180 years ago [6]. Filtering procedures, such as trabeculectomy, or drainage implant surgery, has been used extensively in treatment of glaucoma. Minimally invasive glaucoma surgery could be beneficial for reducing surgical injury, local inflammation and scar formation. However, there are several different procedures which have been used for minimally invasive glaucoma surgery. Each procedure has certain advantages and some disadvantages. It is very important to study the longevity of any glaucoma surgery's outcome. Recently, the different types of minimally invasive glaucoma surgery have been followed clinically [7-10]. Our aim was to develop a safer and more effective surgical procedure. We hoped to overcome any potential causes of the failure of glaucoma surgery and obtain the best surgical outcome for every patient. A minimally invasive surgical technique including bioengineered microfistula, sophisticated implanter and ab-interno surgical procedure was developed by our team $[10,11]$. Our patents (AU\#721915, PCT/AU97/00811, US patent \#6544249) were licensed to a startup company (AqueSys), to commercially develop the technology and AqueSys has recently been acquired by Allergan. It is very likely that our technique will be used worldwide. Such minimally invasive surgery without surgical damage to the conjunctiva could significantly reduce inflammatory reaction and scaring process and provide optimum surgical outcomes. It minimizes conjunctival disturbance and maximizes the ability to visualize the evolution of subconjunctival tissue changes post-operatively. In addition, it creates an opportunity to investigate the consequences of aqueous humor presence in the subconjunctival tissue. We have demonstrated that the conjunctiva and its lymphatics play a critical role in GFS outcomes [11]. Some glaucoma patients may have had long term local drug applications and previous glaucoma surgery. It is important to develop techniques to examine the conjunctiva and its lymphatics before the surgery, and to monitor and modulate subconjunctival tissue properties following GFS. We believe that the answer to such questions could be critical for further improving the outcomes of GFS.

\section{Aqueous flow and glaucoma}

Aqueous humor does not normally contact the subconjunctival tissue. The normal aqueous outflow pathways consist of an intraocular part, exit routes by which aqueous humor leaves the eye, and the return route to the systemic circulation. The major components are the ocular

Correspondence to: Professor Dao-Yi Yu, MD, PhD, Lions Eye Institute, Centre for Ophthalmology and Visual Science, The University of Western Australia, 2 Verdun Street, Nedlands, WA 6009, Australia, Tel: 6189381 0716; E-mail: dyyu@lei.org.au

Key words: Glaucoma, glaucoma filtration surgery, conjunctiva, lymphatics, filtering bleb

Received: March 28, 2016; Accepted: April 22, 2016; Published: April 25, 2016 
immuno privileged system, phagocytic function of the trabecular meshwork, and a fully endothelium lined drainage system. These components play the key roles in the maintenance of stable aqueous flow under normal conditions [11]. The steady-state IOP is developed due to the balance between aqueous production and the resistance to aqueous humor outflow. Elevated IOP in open angle glaucoma is predominantly due to increased outflow resistance. It is generally accepted that in the normal and glaucomatous eye, most of the outflow resistance lies across and within the trabecular meshwork, perhaps in the cribriform region adjacent to the inner wall of Schlemm's canal [12]. It is also known that in glaucoma disease states the aqueous humor quality is altered with greater oxidative stress capacity [13].

\section{Subconjunctival \\ microenvironments \\ and \\ the conjunctival bleb}

GFS allows altered and unfiltered aqueous humor to come into intimate contact with subconjunctival tissue. Aqueous humor in the subconjunctival tissue after GFS unavoidably alters the subconjunctival microenvironment.

It is predicable that a substantial amount of aqueous humor enters into subconjunctival tissue after GFS. Assuming aqueous production by the ciliary epithelium is $\sim 2 \mu \mathrm{L}$ per minute, the volume of aqueous entering into the subconjunctival tissue after GFS could be as much as $3 \mathrm{~mL}$ per day. No doubt that there must be adequate pathways and mechanisms to allow aqueous humor to be removed from the bleb following GFS. To our knowledge, the role of the conjunctiva in the outcomes of GFS has not been systematically reviewed and many critical questions remain unanswered.

Naturally, the pooling of aqueous humor beneath the conjunctiva and/or Tenon's capsule forms a filtering bleb that has been considered a cornerstone of IOP control after GFS [14-19]. Often the bleb appears to be an unstable tissue [20]. Bleb-related complications can be serious and the surgeon has little control over the final appearance of the filtering bleb after surgery [21]. Such complications require very careful management to avoid loss of vision.

It is very important to understand the bleb both structurally and functionally. Aqueous humor enters the subconjunctival tissue and joins the interstitial fluid. Cells and tissue around newly formed pathways are continuously bathed in aqueous humor. Ideally aqueous humor should be removed sooner rather than later. Clinical evidence shows that changes in the number of inflammatory cells and fibroblasts occur in the conjunctiva of eyes at increased risk of GFS failure [22-27] and it is possible that this may influence the wound healing process following surgery. The failure of trabeculectomy is most commonly associated with a fibrotic response at the wound site in the subconjunctival tissue [28]. However, the mechanisms by which aqueous humor drains from the bleb after GFS have not been fully elucidated [29-33]. There is very limited knowledge of the pathways and mechanisms draining the aqueous humor from the bleb.

Without surgical damage to the conjunctiva, we can obtain more reliable information of the conjunctival bleb after microfistula implantation. Figure 1 shows an example from our experimental studies which illustrates detailed and dynamic information from the subconjunctival bleb after GFS, providing clues to the mystery of how aqueous humor enters and drains from the subconjunctival tissue [11]. A video was recorded after fluorescein dye injection into the anterior chamber in a rabbit with a functional bleb after microfistula implantation. A sequence of video frames shows the spread of fluorescein
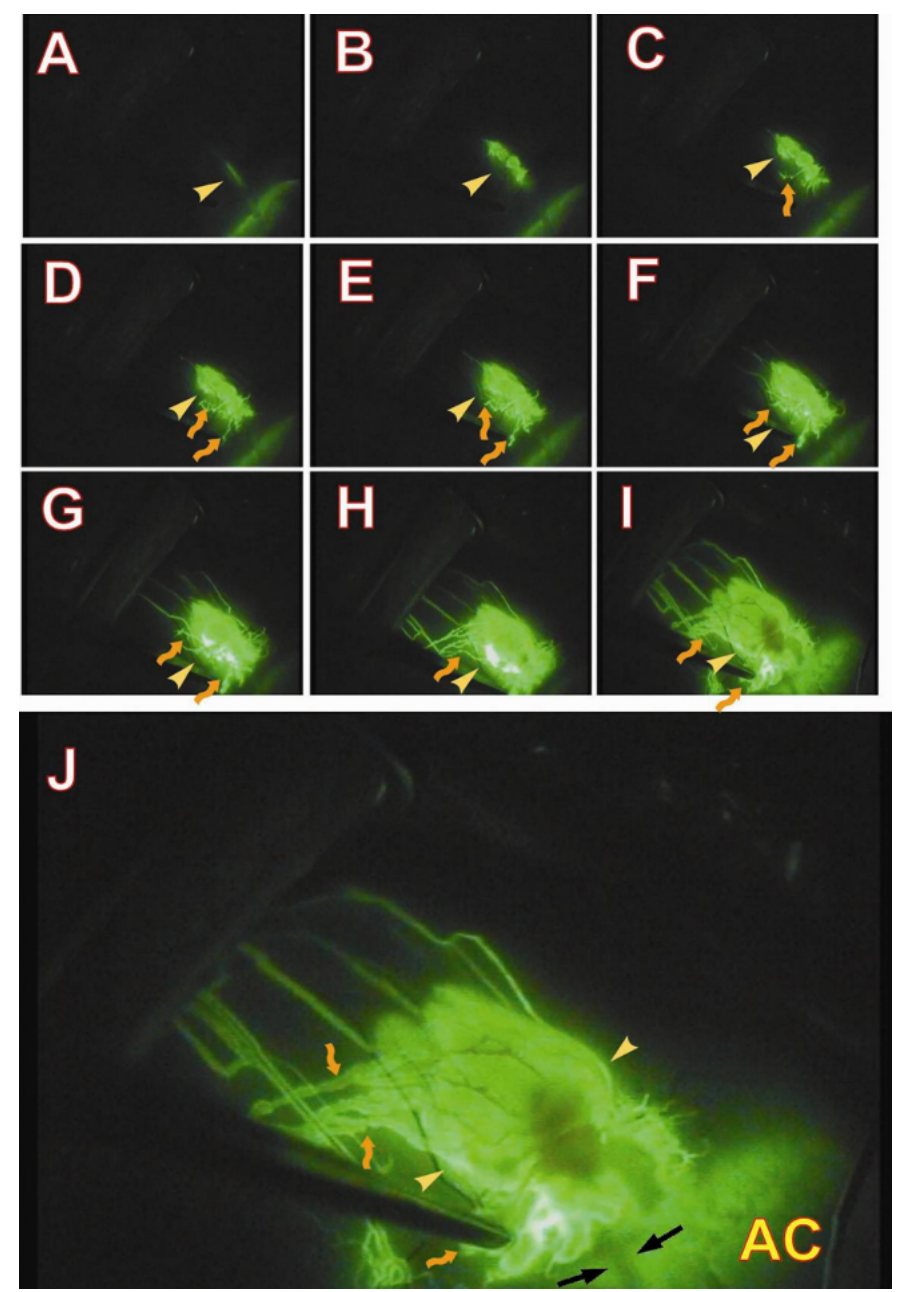

Figure 1. Sequence of video frames (Panels A to I) after injection of fluorescein into the anterior chamber of a rabbit 6 months after microfistula implantation.

Initially a thin line of fluorescein dye appears under the conjunctival tissue directly linking with the scleral channel (Panel A, yellow arrowhead). This thin-line of fluorescein then enlarges and shows a very uneven structure (yellow arrowhead) which is very likely to be initial lymphatics. The fluorescein staining in this line gradually extended in length and area (Panels E to I). More drainage vessels are seen and some of them had a lymphatic appearance (brownish and bent arrow, Panel C to I). In later phases the small vessels are seen along with more extensive filling and increased numbers. The staining within the bleb also increases with time. Panel J shows a magnified view of Panel I. A conjunctival bleb along with a scleral channel (black arrows) and a number of drainage vessels are seen. Some of the drainage vessels appeared even and regular with small calibre and their orientations and appearance are similar to the normal conjunctival and episcleral veins. Other drainage vessels are uneven with larger calibre and their orientations and appearance are similar to normal conjunctival lymphatic vessels (brownish bent arrows). (Modified from Yu, et al.) [11].

staining from the anterior chamber. A thin line of fluorescein dye appeared under the conjunctival tissue linking with the scleral channel, which appeared after fluorescein injection into the anterior chamber. This thin-line of fluorescein then enlarged and showed a very uneven structure along with some initial lymphatics, suggesting that initial lymphatics (lymphatic capillaries) are very close to the scleral exit point of aqueous humor in a functional bleb. The fluorescein staining gradually extended in length and area which may indicate an associated diffusion process. Lymphatic vessels become more extended as more lymphatic vessels filled. Initially the smaller lymphatics were difficult to identify due to their small size and some diffuse leakage. However, in later phases the small lymphatics were clearly evidenced along with 
more extensive filling and increased numbers, and also enlarged with time within the bleb. Now we know that in addition to diffused aqueous humor the bleb also contains a number of initial lymphatics and that these vessels drain aqueous humour forming an important part of the "active" drainage pathways. These "active" drainage pathways appear to play an important role in removal of aqueous fluid from the subconjunctival tissue, although diffusion from the bleb may still be a factor. A conjunctival bleb along with a scleral channel and a number of drainage vessels were apparent. Some of the vessels appeared even and regular with small calibre and their orientations and appearance are similar to the normal conjunctival and episcleral veins. Drainage lymphatics are uneven with larger calibre and their orientation and appearance were similar to normal conjunctival lymphatic vessels.

We have demonstrated the role of lymphatics in drainage of fluid from subconjunctival tissue. Trypan blue was injected into the subconjunctival tissue to create interstitial tissue fluid which was then drained through the lymphatics using a technique similar to that previously described $[34,35]$. A tiny amount of sterile trypan blue was injected using a 32-gauge needle to form a small subconjunctival blister just below the conjunctival epithelium. The pool of trypan blue was observed in order to evaluate if it remained stagnant, meaning lack of lymphatic drainage, or if it spread away from the blister forming distinct branch-like tributaries indicating the existence of lymphatic drainage. The distribution and appearance of normal conjunctival lymphatics in the monkey are shown in Figure 2. After sufficient injection of trypan blue to create a blister in the subconjunctival space the dye rapidly appeared in the most favourable lymphatic channels. The dye then spread throughout the lymphatic vascular tree to reach the major collecting lymphatics (2B). Some backfilling of the smaller initial lymphatics was also evident (2F). Only a small blister of trypan blue was required in the monkey.

Furthermore, we have also demonstrated the conjunctival lymphatics distribution in rabbits and monkeys. We identified that both initial and collecting lymphatics are present in the conjunctiva using the enzyme histochemistry, immunohistochemistry and isolated lymphatics. These initial lymphatics are generally similar to those in other organs. Interstitial tissue fluid, solutes, cells and particulate matter from the conjunctival tissue enter the initial lymphatics. Initial lymphatics have also been termed terminal lymphatics or lymphatic capillaries. These are the most distal structures in the lymphatic system. They vary in size and shape, and are typically blind-ended tubes with variable anastomoses with other initial or collecting lymphatics. Initial lymphatics have very thin walls comprised of non-fenestrated endothelial cells, incomplete basement membrane, and are devoid of muscle cells or pericytes.

We have developed a minimally invasive glaucoma surgery along with bioengineered microfistula and ab-interno approach which can significantly improve glaucoma surgical outcomes. We have also enhanced our knowledge from detailed experimental investigations of drainage mechanisms of aqueous humor in the subconjunctival tissue. We would like to translate our research discoveries of aqueous humor drainage mechanisms in the subconjunctival tissue for clinical diagnosis and further treatment by developing non-invasive and label-free imaging techniques to monitor each patient's conjunctival lymphatics before the operation and the conjunctival bleb after GFS. It can be expected that GFS success rate will be significantly increased if such techniques can be employed. Furthermore, if we could develop new therapeutics to modulate the function of conjunctival lymphatics, we could potentially fine tune lymphatic and hence bleb drainage to
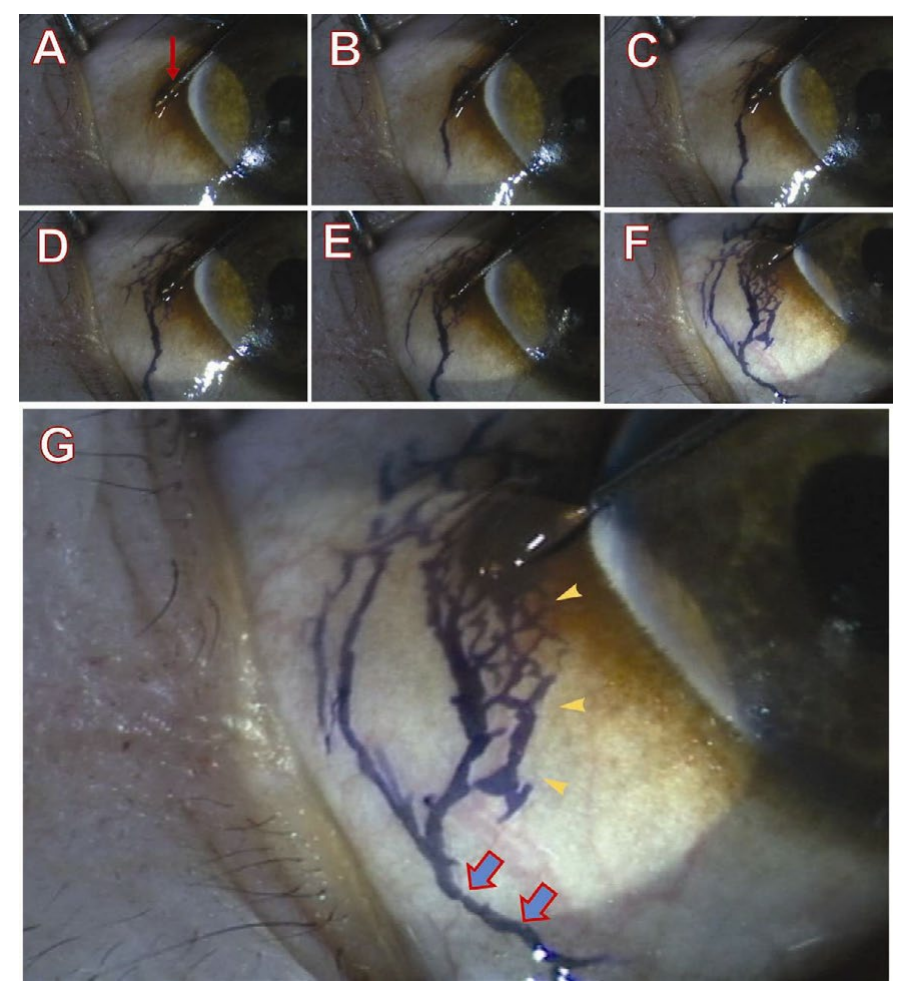

Figure 2. Normal conjunctival lymphatics in the monkey demonstrated by subconjunctival trypan blue injection.

Series of video frames (Panels A to F) taken from a normal monkey to show the local distribution of lymphatics after trypan blue injection. Panel A shows a needle (red arrow) inserted into the superficial layer of the conjunctiva close to the limbus before injection. A small amount of trypan blue was injected into subconjunctival tissue; some conjunctival lymphatic vessels are evident (Panel B). When the amount of trypan blue injection is gradually increased, more extensive lymphatics are filled (Panels B to F). At higher magnification (Panel G) initial lymphatics can be identified (yellow arrowheads) along with larger structures forming the collecting lymphatics (Blue arrows). The distribution of conjunctival lymphatic vessels is clearly different to that of the conjunctival blood vessels). (Modified from $\mathrm{Yu}$, et al.) [11].

more effectively remove the aqueous humor from subconjunctival tissue. It is unavoidable that some glaucoma patients have had long term pharmacological treatments with eye drops containing various substances and/or previous surgical procedures before our procedure, which tend to increase the risk of GFS failure. We would like to share our concepts and preliminary work to improve the monitoring and modulation of subconjunctival tissue and lymphatics after GFS so as to improve the predictability of GFS outcomes.

\section{Clinical monitoring of the subconjunctival microenvi- ronment}

Unlike blood, lymph flow is colorless and the lymphatic vessel wall is very thin. We do not visualize lymphatics in the conjunctiva in normal conditions. Although we use fluorescence and trypan blue dyes to illustrate the presence of conjunctival lymphatics and their roles in the drainage aqueous humor from subconjunctival tissue experimentally, these dyes should be avoided clinically.

Fortunately, non-invasive and label-free techniques, such as optical coherence tomography (OCT) and confocal laser scanning ophthalmoscope (CLSO) have been widely used in the ophthalmology $[10,36]$. Using CLSO and the Heidelberg Rostock Cornea Module technique we have successful imaged optical sections of conjunctival 
bleb (Figure 3). From these sections, we are able to visualize the structure of the subconjunctival tissue and filtration bleb at a microscopic level. But we cannot identify exactly which structure is representing the lymphatics. Advances in OCT techniques may help to identify the lymphatics. The development of OCT has revolutionized ophthalmology by providing a rapid, simple, and non-invasive method to assess retinal structure at the microscopic level [37]. Recently, the development of OCT-angiography (OCT-A) has opened up exciting new avenues for label-free 3D clinical imaging of retinal capillaries, as demonstrated in studies using either commercially available or custom-built systems [38-43]. The OCT-A has successfully obtained retinal capillary and microvasculature images using signals from moving red blood cells. An interesting question is whether we can utilize specific features of the conjunctival lymphatics to identify it clinically. Conjunctival lymphatics have a specific pattern and distribution as we have described $[11,44,45]$. Figure 4 illustrates
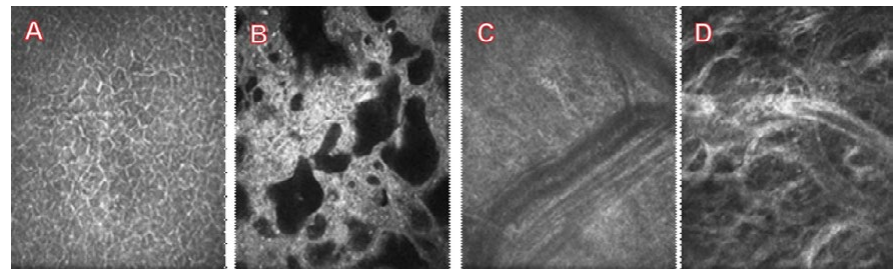

Figure 3. Confocal scanning laser microscopy optical sections from a functional bleb of a patient after trabeculectomy. (A) Conjunctival epithelial cells, (B) Cyst-like irregular shape dark space, (C) Small arterioles and venules and (D) Swelling tissue.
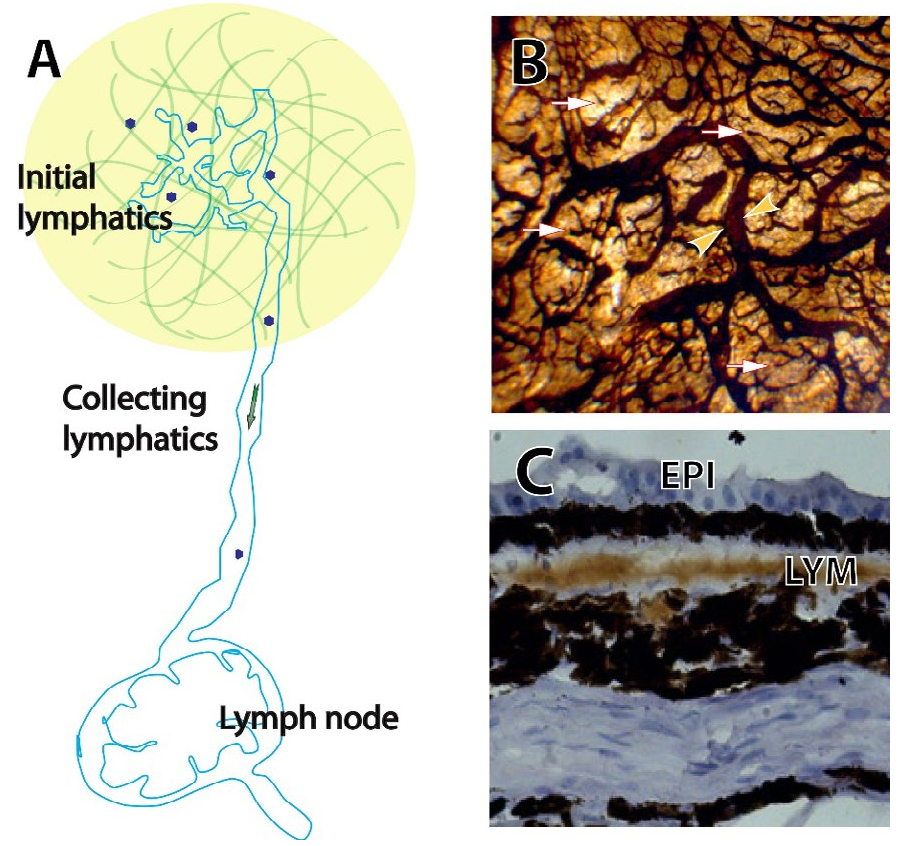

Figure 4. Initial lymphatics, collecting lymphatics and the lymph node. (A) Schematic figure of lymphatic system, including initial lymphatic vessels, collecting lymphatic vessel and the draining lymph node.

(B) Initial lymphatic vessel and collecting lymphatic vessel from normal monkey conjunctival lymphatics Flat mounted conjunctiva which was stained by enzyme histochemistry (5'-nucleotidase). The conjunctiva has relatively dense lymphatic network. Note that the blind-ended tubes at the initial lymphatics (white arrows) and collecting lymphatics (yellow arrowheads) which have a larger and more uneven calibre located in the deeper layer. (C) Cryosection of conjunctiva which was stained by immunochemistry (VEGFR-3) shows conjunctival lymphatics (LYM) under the conjunctival epithelium (EPI). (Modified from Yu, et al.) [11,45] the conjunctival lymphatics which consists of lymphatic capillaries and pre-collectors. The conjunctiva has relatively dense lymphatic network. The blind-ended tubes at the initial lymphatics and collecting lymphatics have a larger and more uneven caliber located in the deeper layer. The conjunctival lymphatics have relatively large size and irregular shape which is distinctly different compared to blood vessels. In addition, initial lymphatics are located under conjunctival epithelium. It may be possible to use OCT techniques to image the conjunctival lymphatics identified by an interconnected large lumen network and the characteristics described above without the presence of moving red blood cells. Label-free optic lymphangiography has been reported by an auto segmentation method applied to OCT to visualize skin lymphatic vessels [46].

\section{Modulation of conjunctival lymphatic capillaries}

The outcomes of GFS can be further improved if conjunctival lymphatic capillaries could be functionally modulated. Aqueous humor after GFS will continuously enter subconjunctival tissue joining interstitial fluid. Lymph vessels are required for carrying that fluid, interstitial proteins, peptides, and cells to the lymph nodes and back to the blood circulation (Figure 4). Initial lymphatic vessels (also called lymphatic capillaries) are present in the conjunctiva in human, rabbits and primates $[11,44]$. An important result from our experimental work is that lymphatic capillaries are very close to the aqueous exit point outside of the sclera in the functional bleb [11]. We still do not know whether these lymphatic capillaries were pre-existing or newly formed by an amoeboid migration of endothelial cells from initial lymphatic vessels or lymphangiogenesis. The endothelial cells in the initial lymphatics are able to undertake amoeboid migration and even phagocytosis [47]. Lymphatic capillaries consist of a single layer of overlapping and interdigitated endothelial cells. These overlapped endothelial cells are joined by the junctional protein VE-cadherin. The abluminal part of the initial lymphatics is connected to the elastic fibers in the surrounding ECM via so-called anchoring filaments, consisting of collagen VII, a connection made via the transmembrane integrin and focal adhesion kinase $[47,48]$. Such a structure has been described as flaps and initial valves allowing one-way passage of cells, fluid, and protein.

Whether "pre-lymphatic channels" with low resistance pathways exist in the normal subconjunctival tissue, as found in the skin and muscle is another interesting question. Interestingly, the integrated effect of spontaneous motion could induce volumetric contraction of the entire initial lymphatics. To change lymphatic lumen volume of a collapsed lymphatic requires tensile tissue stress. A distended lymphatic requires compressive tissue stress to reduce lymphatic volume. The 'tissue pump' and initial lymphatics enable lymph formation and transport. The overlapping junctions may thus constitute a valve system that prevents retrograde flow from the lymphatic capillary back into the interstitium. There are complex mechanisms involved the removing the aqueous humor from subconjunctival tissues by the initial lymphatics. Knowledge of these mechanisms will help us to improve this draining process.

\section{Modulation of conjunctival lymphatic pre-collectors}

We have demonstrated that collecting lymphatics are present in the conjunctiva. The lymphatic valve can be seen histologically in a flatmount of a human conjunctiva stained with 5'-nucleotidase and also in an in vivo monkey after GFS and in an isolated perfused preparation $[11,44]$. These luminal unidirectional valves are closely spaced creating 


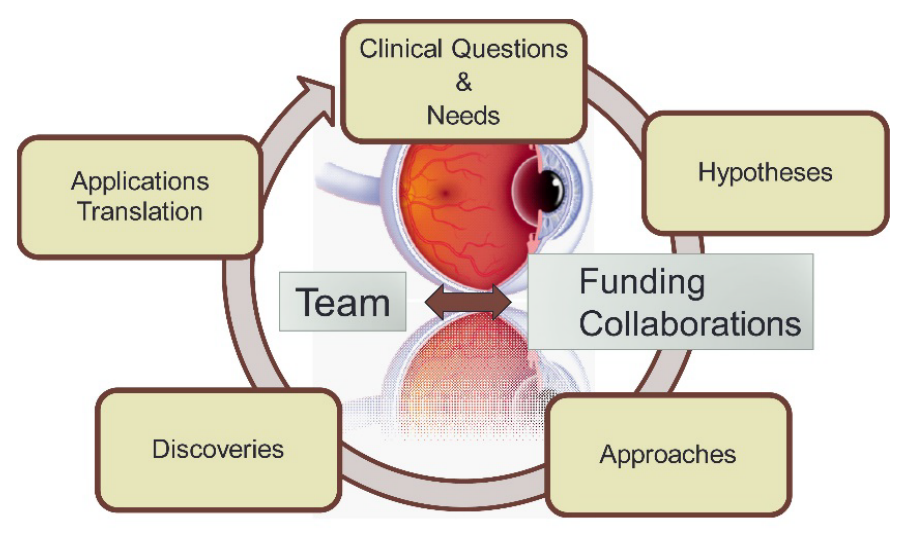

Figure 5. Schematic illustration of translational research

segmented collecting lymphatics. These segments in the collecting vessel form a lymphangion. Segmentation, unidirectional valves and muscular wall are important features of lymphatics. The function of the lymphangion has an intrinsic contractile mechanism and undergoes rhythmical, phasic constrictions to propel the lymph centrally. An isolated perfused conjunctival lymphatic vessel system has been established in our group using methods previously used on isolated retinal arterioles and veins [49-55]. To determine the performance of the collecting lymphatics, we dissected, cannulated and perfused the collecting lymphatics from fresh conjunctiva and studied them. As the importance of the lymphatic system in the human body becomes increasingly evident, novel research effort will drive the need for improved models to study lymphatic biology. Using this preparation, possible modulations of collecting lymphatics by chemicals, drugs and other factors could also be tested. Hopefully, we can find effective compounds which could modulate the function of the conjunctival lymphatic pre-collectors after GFS if required.

\section{Summary}

Translation research is one of the most important aspects of medical research. Our team is focusing on some common and critical questions and needs in the field of ophthalmology. We need to carefully consider our hypotheses, take optimum approaches and perform the appropriate experimental studies. There are exciting opportunities to discover the answers to key questions as the clinical data from the expanding use of this novel GFS approach becomes available. A collaborative approach with industry partners may well provide the most efficient way forward. Translation of new discoveries into clinical practice is not the end of the process. Feedback from clinical use would provide the best pathway to optimizing devices and techniques to produce the optimum outcome for each and every patient. This process (Figure 5) could be long term with many challenges. Therefore, we should consider new questions and challenges raised in clinical applications. We will continue our efforts to answer these new questions and challenges. We propose to collaborate with experts in OCT and lymphatic fields to develop the techniques required to further improve surgical outcomes in GFS.

\section{References}

1. Quigley HA (1996) Number of people with glaucoma worldwide. Br J Ophthalmol 80: 389-393. [Crossref]

2. Lütjen-Drecoll E, Futa R, Rohen JW (1981) Ultrahistochemical studies on tangential sections of the trabecular meshwork in normal and glaucomatous eyes. Invest Ophthalmol Vis Sci 21: 563-573. [Crossref]

3. Lütjen-Drecoll E (1999) Functional morphology of the trabecular meshwork in primate eyes. Prog Retin Eye Res 18: 91-119. [Crossref]
4. Brubaker RF (2003) Targeting outflow facility in glaucoma management. Surv Ophthalmol 48 Suppl 1: S17-20. [Crossref]

5. Tamm ER (2010) Functional Morphology of the Trabecular Meshwork. Encyclopedia of the Eye 157-163.

6. Ritch R, Shields MB, Krupin T (1989) The Glaucomas St Louis: The C.V. Mosby Company. Pp: 1-748.

7. Neiweem AE, Bussel II, Schuman JS, Brown EN, Loewen NA, et al. (2016) Glaucoma Surgery Calculator: Limited Additive Effect of Phacoemulsification on Intraocular Pressure in Ab Interno Trabeculectomy. PLoS One 11: e0153585. [Crossref]

8. Xin C, Chen X, Shi Y, Li M, Wang H, et al. (2016) One-year interim comparison of canaloplasty in primary open-angle glaucoma following failed filtering surgery with primary canaloplasty. Br J Ophthalmol. [Crossref]

9. Mandal AK, Pehere N (2016) Early-onset glaucoma in Axenfeld-Rieger anomaly: longterm surgical results and visual outcome. Eye (Lond). [Crossref]

10. Samples JR, Ahmed IIK (2014) Surgical Innovations in Glaucoma. In: Springer Science+Business Media, New York.

11. Yu DY, Morgan WH, Sun X, Su EN, Cringle SJ, et al. (2009) The critical role of the conjunctiva in glaucoma filtration surgery. Prog Retin Eye Res 28: 303-328. [Crossref]

12. Bill A (1975) Editorial: The drainage of aqueous humor. Invest Ophthalmol 14: 1-3. [Crossref]

13. Ferreira SM, Lerner SF, Brunzini R, Evelson PA, Llesuy SF (2004) Oxidative stress markers in aqueous humor of glaucoma patients. Am J Ophthalmol 137: 62-69. [Crossref]

14. Streilein JW (1995) Immunological non-responsiveness and acquisition of tolerance in relation to immune privilege in the eye. Eye (Lond) 9: 236-240. [Crossref]

15. Streilein JW (2003) Ocular immune privilege: therapeutic opportunities from an experiment of nature. Nat Rev Immunol 3: 879-889. [Crossref]

16. Abrams GW, Thomas MA, Williams GA, Burton TC (1986) Management of postoperative suprachoroidal hemorrhage with continuous-infusion air pump. Arch Ophthalmol 104: 1455-1458. [Crossref]

17. D'Ermo F, Bonomi L (1973) Trabeculectomy. Results in the treatment of glaucomas. Ophthalmologica 166: 311-320. [Crossref]

18. D'Ermo F, Bonomi L, Doro D (1979) A critical analysis of the long-term results of trabeculectomy. Am J Ophthalmol 88: 829-835. [Crossref]

19. Levene RZ (1984) Glaucoma filtering surgery: factors that determine pressure control Ophthalmic Surg 15: 475-483. [Crossref]

20. Classen L, Kivelä T, Tarkkanen A (1996) Histopathologic and immunohistochemical analysis of the filtration bleb after unsuccessful glaucoma seton implantation. Am J Ophthalmol 122: 205-212. [Crossref]

21. Azuara-Blanco A, Katz LJ (1998) Dysfunctional filtering blebs. Surv Ophthalmol 43: 93-126. [Crossref]

22. Broadway D, Grierson I, Hitchings R (1994) Racial differences in the results of glaucoma filtration surgery: are racial differences in the conjunctival cell profile important? Br J Ophthalmol 78: 466-475. [Crossref]

23. Sherwood MB, Grierson I, Millar L, Hitchings RA (1989) Long-term morphologic effects of antiglaucoma drugs on the conjunctiva and Tenon's capsule in glaucomatous patients. Ophthalmology 96: 327-335. [Crossref]

24. Broadway D, Grierson I, O'Brien C, Hitchings RA (1993) The effect of topical antiglaucoma medication on the conjunctival cell profile and the results of trabeculectomy. Invest Ophthalmol Vis Sci 34: 724. [Crossref]

25. Peiffer RL, Popovich KS, Nichols DA (1990) Long-term comparative study of the Schocket and Joseph glaucoma tube shunts in monkeys. Ophthalmic Surg 21: 55-59. [Crossref]

26. Broadway DC, Grierson I, O'Brien C, Hitchings RA (1994) Adverse effects of topical antiglaucoma medication. I. The conjunctival cell profile. Arch Ophthalmol 112: 14371445. [Crossref]

27. Broadway DC, Grierson I, O'Brien C, Hitchings RA (1994) Adverse effects of topical antiglaucoma medication. II. The outcome of filtration surgery. Arch Ophthalmol 112: 1446-1454. [Crossref]

28. Hitchings RA, Grierson I (1983) Clinico pathological correlation in eyes with failed fistulizing surgery. Trans Ophthalmol Soc U K 103 : 84-88. [Crossref] 
29. Herschler J, Claflin AJ, Fiorentino G (1980) The effect of aqueous humor on the growth of subconjunctival fibroblasts in tissue culture and its implications for glaucoma surgery. Am J Ophthalmol 89: 245-249. [Crossref]

30. Radius RL, Herschler J, Claflin A, Fiorentino G (1980) Aqueous humor changes after experimental filtering surgery. Am J Ophthalmol 89: 250-254. [Crossref]

31. Watkins PH Jr, Brubaker RF (1978) Comparison of partial-thickness and full-thickness filtration procedures in open-angle glaucoma. Am J Ophthalmol 86: 756-761. [Crossref]

32. Maumenee AE (1960) External filtering operations for glaucoma: the mechanism of function and failure. Trans Am Ophthalmol Soc 58: 319-328. [Crossref]

33. Teng CC, Chi HH, Katzin HM (1959) Histology and mechanism of filtering operations. Am J Ophthalmol 47: 16-33. [Crossref]

34. Sugar HS, Riazi A, Schaffner R (1957) The bulbar conjunctival lymphatics and their clinical significance. Trans Am Acad Ophthalmol Otolaryngol 61: 212-223. [Crossref]

35. Seetner A, Morin JD (1979) Healing of trabeculectomies in rabbits. Can J Ophthalmol 14: 121-125. [Crossref]

36. Guthoff RF, Baudouin C, Stave J (2006) Atlas of Confocal Laser Scanning In-vivo Microscopy in Ophthalmology - Principles and Applications in Diagnostic and Therapeutic Ophthalmology, Springer, Verlag Berlin Heidelberg Germany.

37. Huang D, Swanson EA, Lin CP, Schuman JS, Stinson WG, et al. (1991) Optica coherence tomography. Science 254: 1178-1181. [Crossref]

38. Mahmud MS, Cadotte DW, Vuong B, Sun C, Luk TW, et al. (2013) Review of speckle and phase variance optical coherence tomography to visualize microvascular networks. J Biomed Opt 18: 50901. [Crossref]

39. Yu PK, Balaratnasingam C, Xu J, Morgan WH, Mammo Z, et al. (2015) Label-Free Density Measurements of Radial Peripapillary Capillaries in the Human Retina. PLoS One 10: e0135151. [Crossref]

40. Xu J, Han S, Balaratnasingam C, Mammo Z, Wong KS, et al. (2015) Retinal angiography with real-time speckle variance optical coherence tomography. $\mathrm{Br} J$ Ophthalmol 99: 1315-1319. [Crossref]

41. Chan G, Balaratnasingam C, Xu J, Mammo Z, Han S, et al. (2015) In vivo optical imaging of human retinal capillary networks using speckle variance optical coherence tomography with quantitative clinico-histological correlation. Microvasc Res 100: 3239. [Crossref]

42. Mammo Z, Balaratnasingam C, Yu P, Xu J, Heisler M, et al. (2015) Quantitative Noninvasive Angiography of the Fovea Centralis Using Speckle Variance Optical Coherence Tomography. Invest Ophthalmol Vis Sci 56: 5074-5086. [Crossref]
43. Tan PE, Balaratnasingam C, Xu J, Mammo Z, Han SX, et al. (2015) Quantitative Comparison of Retinal Capillary Images Derived By Speckle Variance Optica Coherence Tomography With Histology. Invest Ophthalmol Vis Sci 56: 3989-3996. [Crossref]

44. Guo W, Zhu Y, Yu PK, Yu X, Sun X, et al. (2012) Quantitative study of the topographic distribution of conjunctival lymphatic vessels in the monkey. Exp Eye Res 94: 90-97. [Crossref]

45. Knepper PA, Samples JR (2016) Glaucoma Research and Clinical Advances 2016 to 2018 Netherlands: Kugler Publications, Netherlands.

46. Yousefi S, Qin J, Zhi Z, Wang RK (2013) Label-free optical lymphangiography: development of an automatic segmentation method applied to optical coherence tomography to visualize lymphatic vessels using Hessian filters. J Biomed Opt 18: 86004. [Crossref]

47. Schmid-Schönbein GW (1990) Microlymphatics and lymph flow. Physiol Rev 70: $987-$ 1028. [Crossref]

48. Wiig H, Swartz MA (2012) Interstitial fluid and lymph formation and transport: physiological regulation and roles in inflammation and cancer. Physiol Rev 92: 10051060. [Crossref]

49. Su EN, Cringle SJ, Yu DY (2007) Vasoconstrictive effects of sodium fluorescein on retinal vessels is increased by light exposure. Curr Eye Res 32: 77-81. [Crossref]

50. Yu DY, Cringle SJ, Su EN, Yu PK (2005) Sphincter activity in retinal arterioles feeding the deeper capillary layer in pig. Curr Eye Res 30: 781-787. [Crossref]

51. Yu DY, Su EN, Cringle SJ, Yu PK (2003) Isolated preparations of ocular vasculature and their applications in ophthalmic research. Prog Retin Eye Res 22: 135-169. [Crossref]

52. Yu DY, Su EN, Cringle SJ, Schoch C, Percicot CP, et al. (2001) Comparison of the vasoactive effects of the docosanoid unoprostone and selected prostanoids on isolated perfused retinal arterioles. Invest Ophthalmol Vis Sci 42: 1499-1504. [Crossref]

53. Yu DY, Su EN, Cringle SJ, Alder VA, Yu PK, et al. (1998) Effect of betaxolol, timolol and nimodipine on human and pig retinal arterioles. Exp Eye Res 67: 73-81. [Crossref]

54. Su EN, Yu DY, Alder VA, Cringle SJ, Yu PK (1996) Direct vasodilatory effect of insulin on isolated retinal arterioles. Invest Ophthalmol Vis Sci 37: 2634-2644. [Crossref]

55. Yu DY, Alder VA, Cringle SJ, Su EN, Yu PK (1994) Vasoactivity of intraluminal and extraluminal agonists in perfused retinal arteries. Invest Ophthalmol Vis Sci 35: $4087-$ 4099. [Crossref]

Copyright: (C2016 Yu DY. This is an open-access article distributed under the terms of the Creative Commons Attribution License, which permits unrestricted use, distribution, and reproduction in any medium, provided the original author and source are credited. 